\title{
Fuzzy modeling for chaotic systems via interval type-2 T-S fuzzy model with parametric uncertainty
}

\author{
Goran Hasanifard • Ali Akbar Gharaveisi • \\ Mohammad Ali Vali
}

Received: 4 October 2013/Accepted: 15 January 2014/Published online: 21 February 2014

(C) The Author(s) 2014. This article is published with open access at Springerlink.com

\begin{abstract}
A motivation for using fuzzy systems stems in part from the fact that they are particularly suitable for processes when the physical systems or qualitative criteria are too complex to model and they have provided an efficient and effective way in the control of complex uncertain nonlinear systems. To realize a fuzzy model-based design for chaotic systems, it is mostly preferred to represent them by $\mathrm{T}-\mathrm{S}$ fuzzy models. In this paper, a new fuzzy modeling method has been introduced for chaotic systems via the interval type-2 Takagi-Sugeno (IT2 T-S) fuzzy model. An IT2 fuzzy model is proposed to represent a chaotic system subjected to parametric uncertainty, covered by the lower and upper membership functions of the interval type-2 fuzzy sets. Investigating many well-known chaotic systems, it is obvious that nonlinear terms have a single common variable or they depend only on one variable. If it is taken as the premise variable of fuzzy rules and another premise variable is defined subject to parametric uncertainties, a simple IT2 T-S fuzzy dynamical model can be obtained and will represent many well-known chaotic systems. This IT2 T-S fuzzy model can be used for
\end{abstract}

G. Hasanifard $(\bowtie)$

Department of Control Engineering, College of Engineering, Tehran Science and Research Branch, Islamic Azad University, Tehran, Iran

e-mail: goran.hasanifard@iausdj.ac.ir

A. A. Gharaveisi

Department of Electrical Engineering, Shahid Bahonar

University, Kerman, Iran

e-mail: a_gharaveisi@yahoo.com

M. A. Vali

Department of Mathematics, Shahid Bahonar University,

Kerman, Iran

e-mail: mvali@mail.uk.ac.ir physical application, chaotic synchronization, etc. The proposed approach is numerically applied to the wellknown Lorenz system and Rossler system in MATLAB environment.

Keywords Chaotic systems - Interval type-2 TakagiSugeno fuzzy system - Lower and upper membership functions - Parametric uncertainty - Footprint of uncertainty

\section{Introduction}

A chaotic system is a highly complex dynamic nonlinear system and its response exhibits an excessive sensitivity to the initial conditions. The sensitive nature of chaotic systems is commonly called as the butterfly effect [1]. Chaos theory has been applied to a variety of fields such as physical systems [2-4], chemical reactor [5], secure communications [6-8], etc.

As we know, fuzzy systems and fuzzy control are suitable for processes when qualitative criteria are too complex to be modeled. Moreover, they have provided an appropriated method in controlling complex nonlinear systems (such as chaotic system). Among various kinds of fuzzy control or fuzzy system methods, the T-S fuzzy system is widely accepted as a powerful tool for designing fuzzy controllers [9-12]. The type-1 T-S fuzzy model offers a general framework for system analysis and controller synthesis. Many researchers have used the type-1 fuzzy model for modeling chaotic systems and their applications where parametric uncertainties have not been considered [13-17]. Some of these papers are based on fuzzy modeling for just one kind of chaotic system; for example in [16], fuzzy modeling has been considered for Lorenz system. 
However, as the membership functions of type-1 fuzzy sets have limited capability of capturing uncertainty information, the control problem cannot be handled directly if the chaotic system is subjected to parametric uncertainty. Despite having a name which carries the connotation of uncertainty, researchers in [18-21] have shown that there are limitations in the ability of type-1 fuzzy systems (T1 FSs) to model and minimize the effect of uncertainties, because type-1 fuzzy systems are certain regarding the fact that its membership grades are crisp values.

In recent years, type-2 fuzzy logic systems have received much attention from the control community as a powerful tool for nonlinear control. Researchers in [22-27] have proposed a type-2 fuzzy logic system (FLS) to deal with uncertain grades of membership using type-2 fuzzy sets. Melin and et al. [28] have designed optimal controllers for autonomous mobile robots. Castillo and Melin have reviewed the design and optimization of IT2 fuzzy controllers [29]. Denisse and et al. have combined type-2 fuzzy system and optimization methods [30]. The type-2 FLS can be regarded as a collection of theoretically infinite number of type-1 FLSs. As a result, additional information, including system parametric uncertainty, can be captured by the type- 2 FLS. The superiority of IT2 fuzzy sets over type- 1 on dealing with uncertain grades of membership has been shown in various applications [31-34].

In this paper, to deal with some problems still existing in control of chaotic systems via type- $1 \mathrm{~T}-\mathrm{S}$ fuzzy models, we propose an IT2 T-S fuzzy model based on sector nonlinearity to represent the chaotic system subject to parametric uncertainty covered by the lower and upper membership functions of the interval type-2 fuzzy sets. This model can be used to control and synchronize chaotic systems using type- 2 fuzzy system. The features of proposed method can be explained as following:

Motivated by potential applications in modeling chaotic phenomena such as chaos synchronization, communication, physical theory and system, control of chaotic dynamic has received and increasing interest. Since chaotic systems are inherently complex systems, fuzzy modeling provides an appropriate representation for such systems. Between several kinds of fuzzy methods, we use T-S fuzzy model because of the mathematical analysis simplicity and the fact that it provides an exact representation. For chaotic systems that evolve within a bounded region of the state space and parametric uncertainty, the type- $2 \mathrm{~T}-\mathrm{S}$ fuzzy model can represent the nonlinear dynamics by lower and upper membership functions of interval type-2 fuzzy sets. Also, because of computational burden of defuzzification and type reduction for general type-2 FLS, we employ the IT2 sets to decrease computational demands. So, we use IT2 T-S modeling, because type-2 fuzzy systems are able to model structured uncertainties such as parametric perturbations as FOU in fuzzy rules by choosing an appropriated membership functions. So, the main advantage of proposed method is simplicity in mathematical computations and modeling parametric uncertainties.

The rest of this paper is organized as follows. In section "IT2 T-S fuzzy modeling of chaotic system", the IT2 T-S fuzzy model for well-known chaotic systems will be presented. Numerical example is simulated in section "Numerical results and analyses" and short discussion is given in section "Discussion". Finally, conclusions are drawn in section "Conclusion".

\section{IT2 T-S fuzzy modeling of chaotic system}

To realize a fuzzy model-based design with parametric uncertainty, chaotic systems should first be represented by fuzzy models. For this purpose, the IT2 T-S fuzzy model is proposed since it seems to be appropriate for chaotic systems with parametric uncertainties, unmodeled dynamics or structural variations of the system. Investigating many well-known chaotic systems, we found that the nonlinear terms depend only on one variable or have a single common variable such as Hènon [35], Rossler system [36], Lorenz system [37], Lu system [38], etc. If it is taken as the premise variable and parametric uncertainty, unmodeled dynamics or structural variation of system as the other premise variable of type-2 fuzzy rules, a fuzzy dynamical model can be obtained for representing chaotic systems. It has been observed that all the well-known chaotic systems can be applied in synchronization and secure communication either by a fuzzy driving signal or by a crisp one [39]. In the following, we will show how to represent many wellknown chaotic systems by the IT2 T-S fuzzy model.

Consider an IT2 TS fuzzy model with $p$ rules in the following format [40]. Each rule's antecedent contains IT2 fuzzy sets and the consequent is a differential equation of a linear dynamical system.

Rule $i$ :

$\operatorname{IF} Z_{1}(\mathbf{x}(t))$ is $\widetilde{M}_{1}^{i}$ AND $\cdots$ AND $Z_{q}(\mathbf{x}(t))$ is $\widetilde{M}_{q}^{i}$

THEN :

$\dot{\mathbf{x}}=A_{i} \mathbf{x}(t)+b_{i}$

where $\widetilde{M}_{\alpha}^{i}$ is an IT2 fuzzy set of rules $i$ corresponding to the function $Z_{\alpha}(\mathbf{x}(t)), \alpha=1,2, \ldots, q ; i=1,2, \ldots, p ; q$ is a positive integer; $\mathbf{x}(t) \in R^{n}$ is the system state vector; $A_{i} \in$ $R^{n \times n}$ and $b_{i} \in R^{n \times 1}$ are the known system matrices. The firing strength of the $i$-th rule resides in the following interval sets:

$\widetilde{\omega}_{i}(\mathbf{x}(t))=\left[\omega_{i}^{\mathrm{L}}(\mathrm{x}(t)), \omega_{i}^{\mathrm{U}}(\mathbf{x}(t))\right], \quad i=1,2, \ldots, p$ 
where

$$
\begin{aligned}
& \omega_{i}^{\mathrm{L}}(\mathbf{x}(t))=\prod_{\alpha=1}^{q} \underline{\mu}_{\widetilde{M}_{\alpha}^{i}}\left(Z_{\alpha}(\mathbf{x}(t))\right) \geq 0 \\
& \omega_{i}^{\mathrm{U}}(\mathbf{x}(t))=\prod_{\alpha=1}^{q} \bar{\mu}_{\widetilde{M}_{\alpha}^{i}}\left(Z_{\alpha}(\mathbf{x}(t))\right) \geq 0
\end{aligned}
$$

In which $\omega_{i}^{\mathrm{L}}(\mathbf{x}(t))$ and $\omega_{i}^{\mathrm{U}}(\mathbf{x}(t))$ denote the lower and upper grades of membership, respectively. The functions $\underline{\mu}_{M_{\alpha}^{i}}\left(Z_{\alpha}(\mathbf{x}(t))\right)$ and $\bar{\mu}_{\widetilde{M}_{\alpha}^{i}}\left(Z_{\alpha}(\mathbf{x}(t))\right)$ are the lower and upper membership functions, respectively. The IT2 T-S fuzzy model is defined as follows:

$$
\begin{aligned}
\dot{\mathbf{x}}(t)= & \sum_{i=1}^{p} \omega_{i}^{\mathrm{L}}(\mathbf{x}(t)) \underline{v}_{i}(\mathbf{x}(t))\left(A_{i} \mathbf{x}(t)+b_{i}\right) \\
& +\sum_{i=1}^{p} \omega_{i}^{\mathrm{U}}(\mathbf{x}(t)) \bar{v}_{i}(\mathbf{x}(t))\left(A_{i} \mathbf{x}(t)+b_{i}\right) \\
= & \sum_{i=1}^{p} \omega_{i}(\mathbf{x}(t))\left(A_{i} \mathbf{x}(t)+b_{i}\right)
\end{aligned}
$$

where

$\left\{\begin{array}{l}\omega_{i}(\mathbf{x}(t))=\omega_{i}^{L}(\mathbf{x}(t)) \underline{v}_{i}(\mathbf{x}(t))+\omega_{i}^{U}(\mathbf{x}(t)) \bar{v}_{i}(\mathbf{x}(t)) \geq 0 \\ \forall i, \sum_{i=1}^{p} \omega_{i}(\mathbf{x}(t))=1\end{array}\right.$

In which $\underline{v}_{i}(\mathbf{x}(t)) \geq 0$ and $\bar{v}_{i}(\mathbf{x}(t)) \geq 0$ are nonlinear functions in which $\forall i, \underline{v}_{i}(\mathbf{x}(t))+\bar{v}_{i}(\mathbf{x}(t))=1$. In [41] the functions of $v_{i}(\mathbf{x}(t))$ and $\bar{v}_{i}(\mathbf{x}(t))$ are both defined as 0.5 .

In this paper, some classical chaotic dynamic systems with parametric uncertainty as the following form have been considered:

$\dot{\mathbf{x}}=F(\mathbf{x}) \cdot \mathbf{x}+\Delta F(\mathbf{x})$

where $\mathbf{x}=\left(x_{1}, x_{2}, \ldots, x_{n}\right)^{T} \in R^{n}$ is the state vector, $F(\mathbf{x})$ is $n \times 1$ matrix with nonlinear function and $\Delta \boldsymbol{F}(\mathbf{x})$ is $n \times 1$ uncertain matrix representing the unmodeled dynamics or structural variations of the system which is supposed to be bounded. Two assumptions have been considered to avoid a complicated fuzzy modeling as follows:

Assumption 1 Considering the boundedness of chaotic systems, it is supposed that the fuzzy set is chosen as the region of the system trajectory in the set:

$\left.\Omega=\left\{\mathbf{x}(t) \in \mathrm{R}^{n}: \| \mathbf{x}(t)\right\} \| \leq \beta\right\}$

For chaotic systems, the existence of the parameter $\beta$ is natural.

Assumption 2 Suppose that there is a chaotic system with uncertain parameters, i.e. in Eq. (7), some elements of $\Delta \boldsymbol{F}(\mathbf{x})$ is equal to $\varepsilon_{k} \delta(t) \cdot x_{j}$, where $0<\varepsilon_{k}<\gamma_{k}$ is the amplitude of the white noise $\varepsilon_{k} \delta(t)$.

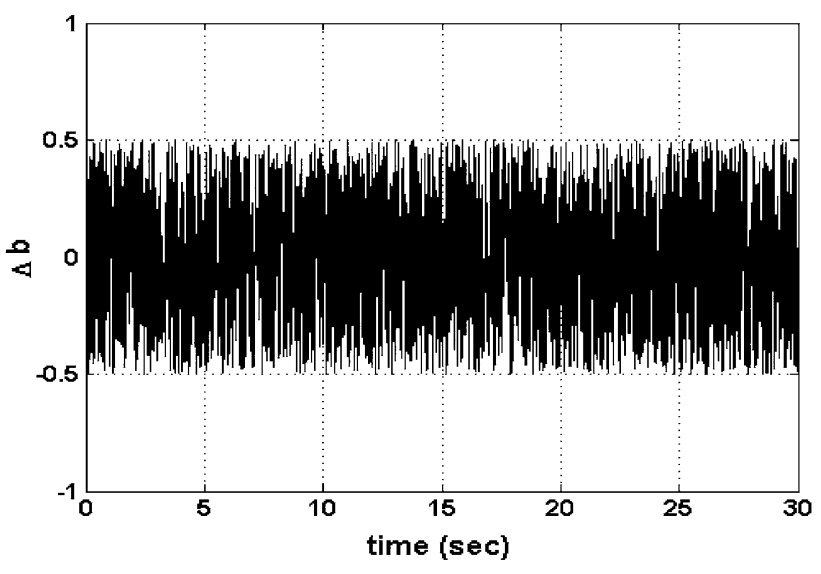

Fig. 1 White noise $\Delta b=0.5 \delta(t)$

\section{Numerical results and analyses}

Although this method is general, for simplicity and less computational effort, only the construction of IT2 T-S fuzzy model for Lorenz system and Rossler system subject to parametric uncertainty will be illustrated, while considering chaotic system subject to uncertain parameter of $\Delta \boldsymbol{F}(\mathbf{x})$ as assumption 1 with known lower and upper bounds.

Lorenz system: with only one uncertain parameter

Consider the following Lorenz system:

$\left\{\begin{array}{l}\dot{x}_{1}=10\left(x_{2}-x_{1}\right) \\ \dot{x}_{2}=28 x_{1}-x_{2}-x_{1} x_{3} \\ \dot{x}_{3}=x_{1} x_{2}-b(t) x_{3}\end{array}\right.$

Suppose that $b(t)$ is the uncertain parameter, i.e., $b(t)=$ $b+\Delta b$ where $b=\frac{8}{3}$ and $\Delta b=\varepsilon_{1} \delta(t)$ is an unknown function, where $\varepsilon_{1}=0.5$ is the amplitude of the white noise, as shown in Fig. 1. Then, the chaotic system is given as follow:

$\left\{\begin{array}{l}\dot{x}_{1}=10\left(x_{2}-x_{1}\right) \\ \dot{x}_{2}=28 x_{1}-x_{2}-x_{1} x_{3} \\ \dot{x}_{3}=x_{1} x_{2}-(b+\Delta b) x_{3}\end{array}\right.$

Comparing Eqs. (7) and (9), we have

$F(\mathbf{x})=\left[\begin{array}{ccc}-10 & 10 & 0 \\ 28 & -1 & -x_{1} \\ 0 & x_{1} & -b\end{array}\right] \quad \Delta F(\mathbf{x})=\left[\begin{array}{c}0 \\ 0 \\ -\Delta b \cdot x_{3}\end{array}\right]$

As mentioned, many researchers have introduced type-1 TS fuzzy model using the sector nonlinearity technique [42] for chaotic system, but type-1 TS fuzzy sets have limited capability of capturing the uncertainty information; 
thus, control problems cannot be handled directly if the chaotic system is subjected to parametric uncertainty.

When $b$ is considered as constant, simulation with initial value $\mathbf{x}(0)=(1,1,1)^{\mathrm{T}}$ gives $x_{1} \in[-2020]$. Choosing $Z_{1}(\mathbf{x}(t))=x_{1} \quad$ as scheduling variable deduces $Z_{1}(\mathbf{x}(t)) \in[-2020]$. Referring to the variables and denoting the membership function as:

$\mu_{M_{1}^{1}}\left(Z_{1}(\mathbf{x}(t))\right)=\frac{20-x_{1}}{40}$

$\mu_{M_{1}^{2}}\left(Z_{1}(\mathbf{x}(t))\right)=1-\mu_{M_{1}^{1}}\left(Z_{1}(\mathbf{x}(t))\right)=\frac{20+x_{1}}{40}$

the following type-1 fuzzy rule is employed to describe the Lorenz system:

Rule $i$ :

$\operatorname{IF} Z_{1}(\mathbf{x}(t))$ is $M_{1}^{i}$

THEN: $\dot{\mathbf{x}}=A_{i} x(t) ; i=1,2$

where $M_{1}^{1}$ and $M_{1}^{2}$ are type-1 fuzzy sets; with

$A_{1}=\left[\begin{array}{ccc}-10 & 10 & 0 \\ 28 & -1 & 20 \\ 0 & -20 & \frac{8}{3}\end{array}\right], \quad A_{2}=\left[\begin{array}{ccc}-10 & 10 & 0 \\ 28 & -1 & -20 \\ 0 & 20 & \frac{8}{3}\end{array}\right]$

The type-1 TS fuzzy model is defined as follows:

$\dot{\mathbf{x}}=\sum_{i=1}^{2} \hat{\omega}_{i}(\mathbf{x}(t))\left(A_{i} \mathbf{x}(t)\right)$

Where the normalized grades of membership are defined as:

$\hat{\omega}_{i}(\mathbf{x}(t))=\frac{\mu_{M_{1}^{i}}\left(Z_{1}(\mathbf{x}(t))\right)}{\mu_{M_{1}^{1}}\left(Z_{1}(\mathbf{x}(t))\right)+\mu_{M_{1}^{2}}\left(Z_{1}(\mathbf{x}(t))\right)}, \quad i=1,2$.

It should be noted that $b$ is assumed to be a constant. So, as mentioned above, the type-1 fuzzy model cannot consider $b$ as an uncertain parameter. Instead, an IT2 T-S fuzzy model is proposed.

Now, we consider $b$ as an uncertain parameter with a perturbation $\Delta b$ where $\Delta b$ satisfies assumption 1, i.e. $\Delta b \in[-0.50 .5]$. With initial value $\mathbf{x}(0)=(1,1,1)^{\mathrm{T}}$, simulation gives $x_{1} \in\left[\begin{array}{ll}-20 & 20]\end{array}\right]$ and $x_{3} \in\left[\begin{array}{ll}1 & 48\end{array}\right]$. Choosing $Z_{1}(\mathbf{x}(t))=x_{1}$ and $Z_{2}(\mathbf{x}(t))=\Delta b \cdot x_{3}$ as scheduling variables gives $Z_{1}(\mathbf{x}(t)) \in\left[\begin{array}{ll}-20 & 20\end{array}\right]$ and $Z_{2}(\mathbf{x}(t)) \in\left[\begin{array}{ll}-24 & 24\end{array}\right]$, i.e. the lower and upper bounds of $Z_{1}(\mathbf{x}(t))$ and $Z_{2}(\mathbf{x}(t))$ can be set. Then, an IT2 T-S fuzzy model with four rules of the following format is employed to describe the Lorenz system subject to uncertainty of parameters:
Rule 1:

$\operatorname{IF} Z_{1}(\mathbf{x}(t))$ is $\widetilde{M}_{1}^{1}$ AND $Z_{2}(\mathbf{x}(t))$ is $\widetilde{M}_{2}^{1}$

THEN: $\dot{\mathbf{x}}=A_{1} \mathbf{x}(t)+b_{1}$

Rule 2:

$\operatorname{IF} Z_{1}(\mathbf{x}(t))$ is $\widetilde{M}_{1}^{2}$ AND $Z_{2}(\mathbf{x}(t))$ is $\widetilde{M}_{2}^{2}$

THEN $\dot{\mathbf{x}}=A_{2} \mathbf{x}(t)+b_{2}$

Rule 3:

$\operatorname{IF} Z_{1}(\mathbf{x}(t))$ is $\widetilde{M}_{1}^{3}$ AND $Z_{2}(\mathbf{x}(t))$ is $\widetilde{M}_{2}^{3}$

THEN $\dot{\mathbf{x}}=A_{3} \mathbf{x}(t)+b_{3}$

Rule 4:

$\operatorname{IF} Z_{1}(\mathbf{x}(t))$ is $\widetilde{M}_{1}^{4}$ AND $Z_{2}(\mathbf{x}(t))$ is $\widetilde{M}_{2}^{4}$

THEN: $\dot{\mathbf{x}}=A_{4} \mathbf{x}(t)+b_{4}$

The IT2 T-S fuzzy model is defined as follows:

$$
\begin{aligned}
\dot{\mathbf{x}}(t) & =\sum_{i=1}^{4}\left(\omega_{i}^{\mathrm{L}}(\mathbf{x}(t)) \underline{v}_{i}(\mathbf{x}(t))+\omega_{i}^{\mathrm{U}}(\mathbf{x}(t)) \bar{v}_{i}(\mathbf{x}(t))\right)\left(A_{i} \mathbf{x}(t)+b_{i}\right) \\
& =\sum_{i=1}^{4} \widetilde{\omega}_{i}(\mathbf{x}(t))\left(A_{i} \mathbf{x}(t)+b_{i}\right)
\end{aligned}
$$

where

$\widetilde{\omega}_{i}(\mathbf{x}(t))=\omega_{i}^{\mathrm{L}}(\mathbf{x}(t)) \underline{v}_{i}(\mathbf{x}(t))+\omega_{i}^{\mathrm{U}}(\mathbf{x}(t)) \bar{v}_{i}(\mathbf{x}(t))$

The lower and upper membership functions are required to satisfy the following inequalities:

$$
\begin{array}{r}
\underline{\mu}_{\widetilde{M}_{1}^{i}}\left(Z_{1}(\mathbf{x}(t))\right) \leq \mu_{M_{1}^{i}}\left(Z_{1}(\mathbf{x}(t))\right) \leq \bar{\mu}_{\widetilde{M}_{1}^{i}}\left(Z_{1}(\mathbf{x}(t))\right) \\
i=1,2,3,4 \\
\underline{\mu}_{\widetilde{M}_{2}^{i}}\left(Z_{2}(\mathbf{x}(t))\right) \leq \mu_{M_{2}^{i}}\left(Z_{2}(\mathbf{x}(t))\right) \leq \bar{\mu}_{\widetilde{M}_{2}^{i}}\left(Z_{2}(\mathbf{x}(t))\right) \\
i=1,2,3,4
\end{array}
$$

It can be deduced from Eqs. (19) and (20) that the lower and upper membership functions form the footprint of uncertainty (FOU) that captures the parametric uncertainty $b$. Any type-1 fuzzy can be reconstructed based on the lower and upper membership functions. As a result, the IT2 T-S fuzzy model is equivalent to infinite number of type-1 fuzzy models. Selecting values as in assumptions 1 and 2 and considering numerical values, it can be determined that the lower and upper bounds of $\mu_{\widetilde{M}_{1}^{i}}\left(Z_{1}(\mathbf{x}(t))\right)$ and $\mu_{\widetilde{M}_{2}^{i}}\left(Z_{2}(\mathbf{x}(t))\right)$ are taken as the lower and upper membership functions of the IT2 T-S fuzzy model. It should be noted that $Z_{1}(\mathbf{x}(t))$ has no uncertain parameters and therefore, lower and upper membership functions are the same. The lower and upper membership functions are defined based on sector nonlinearity in Table 1 . The lower and upper normalized grades of membership for each rule are defined as: 
Table 1 Lower and upper membership functions of Lorenz system Lower membership functions $\quad$ Upper membership functions

$\underline{\mu}_{\widetilde{M}_{1} 1}\left(Z_{1}(\mathbf{x}(t))\right)=\frac{20-x_{1}}{40} \quad \bar{\mu}_{\widetilde{M}_{1}^{1}}\left(Z_{1}(\mathbf{x}(t))\right)=\frac{20-x_{1}}{40}$

$\underline{\mu}_{\widetilde{M}}^{2}{ }_{1}\left(Z_{1}(\mathbf{x}(t))\right)=\frac{20-x_{1}}{40}$

$\bar{\mu} \widetilde{M}_{1}^{2}\left(Z_{1}(\mathbf{x}(t))\right)=\frac{20-x_{1}}{40}$

$\underline{\mu}_{M}{ }_{1}^{3}\left(Z_{1}(\mathbf{x}(t))\right)=\frac{20+x_{1}}{40}$

$\bar{\mu}_{\widetilde{M}_{1}^{3}}\left(Z_{1}(\mathbf{x}(t))\right)=\frac{20+x_{1}}{40}$

$\underline{\mu}_{\mathcal{M}_{1}}\left(Z_{1}(\mathbf{x}(t))\right)=\frac{20+x_{1}}{40}$

$\bar{\mu}{\widetilde{M_{1}^{4}}}\left(Z_{1}(\mathbf{x}(t))\right)=\frac{20+x_{1}}{40}$

$\underline{\mu}_{\widetilde{M}_{2}}\left(Z_{2}(\mathbf{x}(t))\right)=\frac{Z_{2 \max }-\Delta b x_{3}}{Z_{2 \max }-Z_{2 \min }}$ with

$\bar{\mu}_{\widetilde{M}_{2}^{1}}\left(Z_{2}(\mathbf{x}(t))\right)=\frac{Z_{2 \max }-\Delta b x_{3}}{Z_{2 \max }-Z_{2 \min }}$ with

$\Delta b=-0.5$

$\Delta b=0.5$

$\underline{\mu}_{\widetilde{M}_{2}^{2}}{ }_{2}\left(Z_{2}(\mathbf{x}(t))\right)=\frac{Z_{2 \max }-\Delta b x_{3}}{Z_{2 \max }-Z_{2 \min }}$ with

$\bar{\mu}_{\widetilde{M}_{2}^{2}}\left(Z_{2}(\mathbf{x}(t))\right)=\frac{Z_{2 \max }-\Delta b x_{3}}{Z_{2 \max }-Z_{2 \min }}$ with $\Delta b=0.5$

$\Delta b=-0.5$

$\underline{\mu}_{M_{1}^{3}}^{\sim}\left(Z_{2}(\mathbf{x}(t))\right)=\frac{Z_{2 \max }-\Delta b x_{3}}{Z_{2 \max }-Z_{2 \min }}$ with

$\bar{\mu}_{\widetilde{M}_{2}^{3}}\left(Z_{2}(\mathbf{x}(t))\right)=\frac{Z_{2 \max }-\Delta b x_{3}}{Z_{2 \max }-Z_{2 \min }}$ with

$\Delta b=0.5$

$\underline{\mu}_{M_{2}^{4}}\left(Z_{2}(\mathbf{x}(t))\right)=\frac{Z_{2 \max }-\Delta b x_{3}}{Z_{2 \max }-Z_{2 \min }}$ with $\Delta b=-0.5$

$\bar{\mu}_{M_{2}^{4}}\left(Z_{2}(\mathbf{x}(t))\right)=\frac{Z_{2 \max }-\Delta b x_{3}}{Z_{2 \max }-Z_{2 \min }}$ with $\Delta b=0.5$

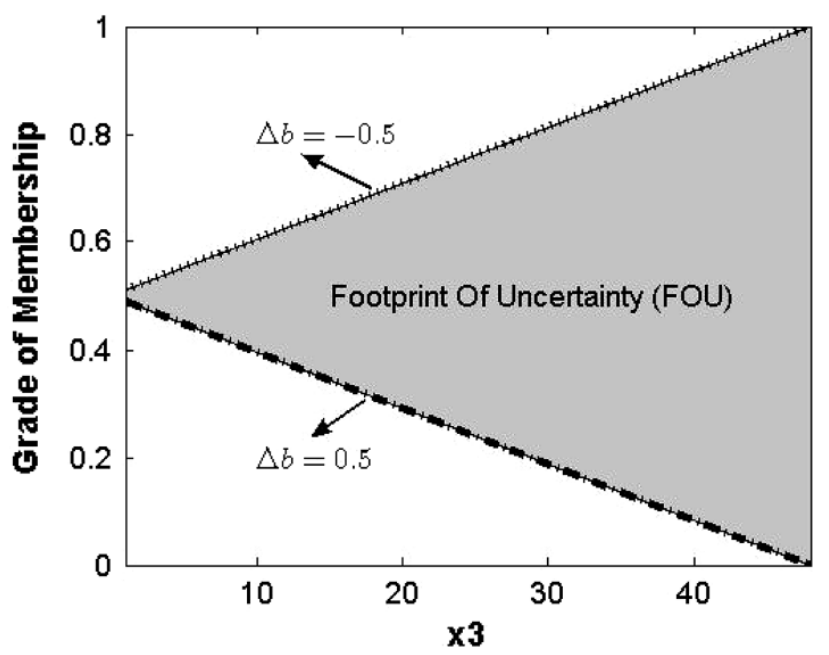

Fig. 2 Plot of $\mu_{\widetilde{M}_{2}^{1}}\left(Z_{2}(\mathbf{x}(t))\right)=\frac{Z_{2 \max }-\Delta b x_{3}}{Z_{2 \max }-Z_{2 \min }}$, lower membership function $\underline{\mu}_{\widetilde{M}_{2}^{1}}\left(Z_{2}(\mathbf{x}(t))\right)$ with $\Delta b=0.5$ (dash-dot line), upper membership function $\bar{\mu}_{\widetilde{M}_{2}^{1}}\left(Z_{2}(\mathbf{x}(t))\right)$ with $\Delta b=-0.5$ (dotted line) and footprint of uncertainty (grey area)

$\omega_{i}^{L}(\mathbf{x}(t))=\underline{\mu}_{\widetilde{M}_{1}^{i}}\left(Z_{1}(\mathbf{x}(t))\right) \cdot \underline{\mu}_{\widetilde{M}_{2}^{i}}\left(Z_{2}(\mathbf{x}(t))\right)$

$\omega_{i}^{\mathrm{U}}(\mathbf{x}(t))=\bar{\mu}{\widetilde{M_{1}^{i}}}^{i}\left(Z_{1}(\mathbf{x}(t))\right) \cdot \bar{\mu}{\widetilde{M_{2}^{i}}}\left(Z_{2}(\mathbf{x}(t))\right)$ for all $i$.

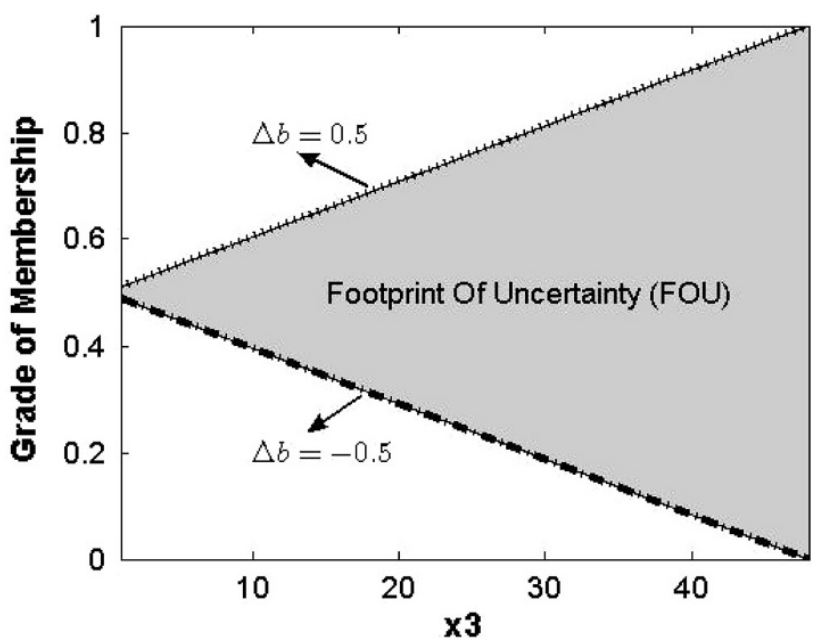

Fig. 3 Plot of $\mu_{\widetilde{M}_{2}^{2}}\left(Z_{2}(\mathbf{x}(t))\right)=\frac{Z_{2 \max }-\Delta b x_{3}}{Z_{2 \max }-Z_{2 \min }}$, lower membership function $\underline{\mu}_{\widetilde{M}_{2}^{2}}\left(Z_{2}(\mathbf{x}(t))\right)$ with $\mathrm{v} \Delta b=-0.5$ (dash-dot line), upper membership function $\bar{\mu}_{\widetilde{M}_{2}^{2}}\left(Z_{2}(\mathbf{x}(t))\right)$ with $\Delta b=0.5$ (dotted line) and footprint of uncertainty (grey area)

The plots of the lower and upper membership functions of the IT2 T-S fuzzy model are shown in Figs. 2 and 3.

Figure 4 shows the difference between fuzzy model and original system or modeling error with $\Delta b=-0.5,0,0.5$

Based on defined FOU in Figs. 2 and 3, the IT2 T-S fuzzy model can be considered as a collection of type-1 T-S fuzzy models. In other words, a type-1 fuzzy model can be defined for every value of $\Delta b$. For example, we have simulated fuzzy model of Lorenz system in the form of eq. (8) for three values of $\Delta b$. As it is evident in Fig. 4, the difference between fuzzy model and original system becomes $<0.5 \times 10^{-4}$ after about $1.5 \mathrm{~s}$, i.e. the fuzzy model can represent the original system. It should be noted that the IT2 TS fuzzy model serves as a mathematical tool to facilitate the design of the IT2 fuzzy controller. In this system, based on IT2 T-S fuzzy model, an IT2 fuzzy controller with four rules can be employed to chaos synchronization and other applications.

Rossler system: with two uncertain parameters

Consider the following Rossler system:

$$
\left\{\begin{array}{l}
\dot{x}_{1}=-x_{2}-x_{3} \\
\dot{x}_{2}=x_{1}+0.2 x_{2} \\
\dot{x}_{3}=a(t)+x_{3}\left(x_{1}-b(t)\right)
\end{array}\right.
$$

Suppose that $a(t)$ and $b(t)$ are the uncertain parameters, i.e. $a(t)=a+\Delta a$ and $b(t)=b+\Delta b$, where $a=0.2$, $b=5$ and $\Delta a=\varepsilon_{1} \delta(t)$ and $\Delta b=\varepsilon_{2} \delta(t)$ are unknown function, where $\varepsilon_{1}=0.1$ and $\varepsilon_{2}=0.1$ are the amplitudes of the white noise as shown in Fig. 5. Then, the chaotic system is given as follows: 

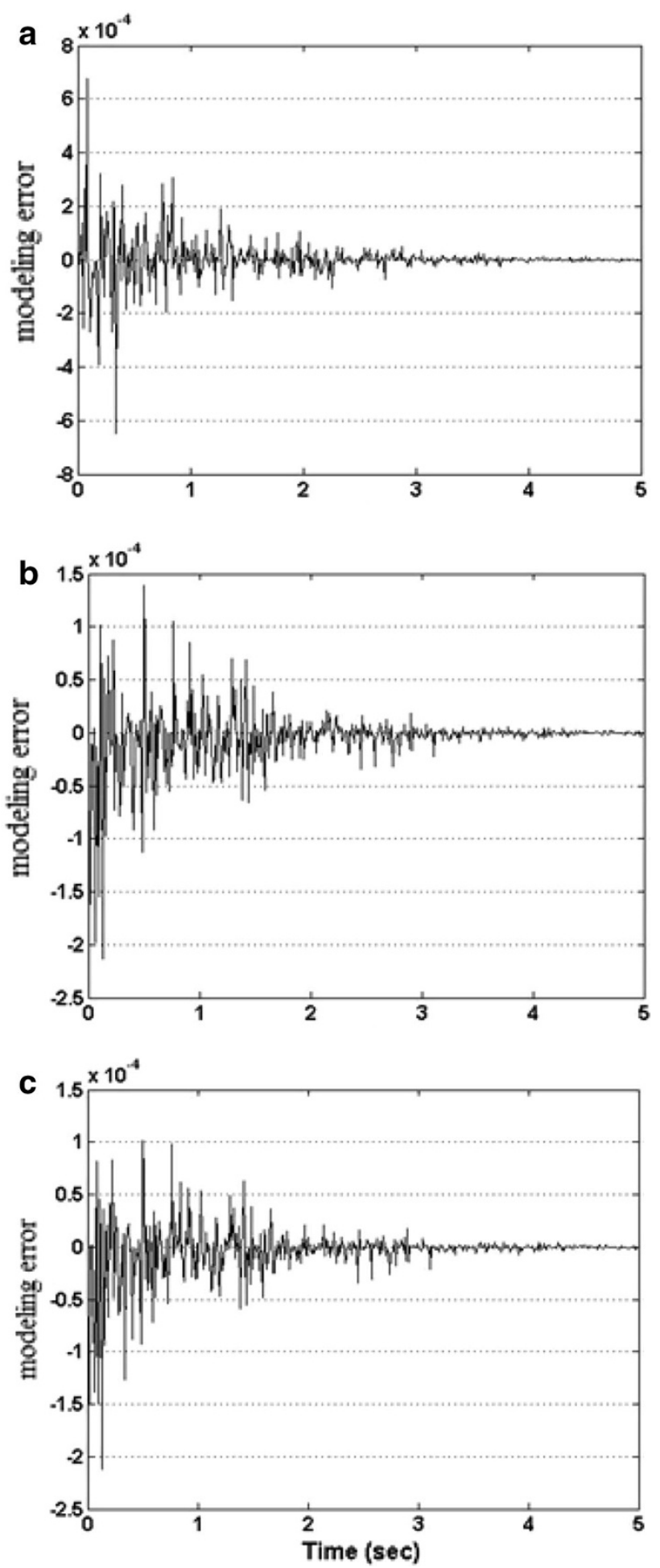

Fig. 4 Difference between fuzzy model and original system of Lorenz system with a $\Delta b=-0.5, \mathbf{b} \Delta b=0$ and $\mathbf{c} \Delta b=0.5$

$$
\left\{\begin{array}{l}
\dot{x}_{1}=-x_{2}-x_{3} \\
\dot{x}_{2}=x_{1}+0.2 x_{2} \\
\dot{x}_{3}=(a+\Delta a)+x_{3}\left(x_{1}-(b+\Delta b)\right)
\end{array}\right.
$$

Comparing Eqs. (7) and (23) we have:

$$
F(\mathbf{x})=\left[\begin{array}{ccc}
0 & -1 & -1 \\
1 & 0.2 & 0 \\
0 & 0 & x_{1}-5
\end{array}\right], \quad \Delta F(\mathbf{x})=\left[\begin{array}{c}
0 \\
0 \\
a(t)-\Delta b \cdot x_{3}
\end{array}\right]
$$

Since membership functions in type-1 fuzzy systems are crisp, type-1 fuzzy model cannot consider $a$ and $b$ as uncertain parameters. Instead, we define an IT2 T-S fuzzy model to conquest this problem. For this purpose, $a$ and $b$ are considered as uncertain parameters with perturbation of $\Delta a$ and $\Delta b$ which satisfy the assumption 1, i.e. $\Delta a \in$ $[-0.10 .1]$ and $\Delta b \in[-0.50 .5]$. It can be seen that $\underline{a} \leq a(t) \leq \bar{a}$ where $\underline{a}=a-\Delta a$ and $\bar{a}=a+\Delta a$ are the constant lower and upper bounds of $a$, respectively.

With initial value $\mathbf{x}(0)=(1,1,1)^{T}$, simulation gives $x_{1} \in\left[\begin{array}{ll}-10 & 10\end{array}\right]$ and $x_{3} \in\left[\begin{array}{ll}0 & 18\end{array}\right]$. Choosing $Z_{1}(\mathbf{x}(t))=$ $x_{1}$ and $Z_{2}(\mathbf{x}(t))=a(t)-\Delta b \cdot x_{3}$ as scheduling variables gives $Z_{1}(\mathbf{x}(t)) \in\left[\begin{array}{lll}-10 & 10\end{array}\right]$ and $Z_{2}(\mathbf{x}(t)) \in[-8.99 .1]$, i.e. the lower and upper bounds of $Z_{1}(\mathbf{x}(t))$ and $Z_{2}(\mathbf{x}(t))$ can be set. Then, an IT2 T-S fuzzy model with four rules of the following format can be employed to describe the Rossler system subject to uncertainty of parameters:

Rule 1:

$\operatorname{IF} Z_{1}(\mathbf{x}(t))$ is $\widetilde{M}_{1}^{1} \mathrm{ANDZ}_{2}(\mathbf{x}(t))$ is $\widetilde{M}_{2}^{1}$

THEN: $\dot{\mathbf{x}}=A_{1} \mathbf{x}(t)+b_{1}$

Rule 2:

$\operatorname{IF} Z_{1}(\mathbf{x}(t))$ is $\widetilde{M}_{1}^{2} \operatorname{ANDZ}_{2}(\mathbf{x}(t))$ is $\widetilde{M}_{2}^{2}$

THEN: $\dot{\mathbf{x}}=A_{2} \mathbf{x}(t)+b_{2}$

Rule 3:

$\operatorname{IF} Z_{1}(\mathbf{x}(t))$ is $\tilde{M}_{1}^{3} \operatorname{AND}_{2}(\mathbf{x}(t))$ is $\tilde{M}_{2}^{3}$

THEN: $\dot{\mathbf{x}}=A_{3} \mathbf{x}(t)+b_{3}$

Rule 4:

$\operatorname{IF} Z_{1}(\mathbf{x}(t))$ is $\widetilde{M}_{1}^{4} \mathrm{AND} Z_{2}(\mathbf{x}(t))$ is $\widetilde{M}_{2}^{4}$

THEN: $\dot{\mathbf{x}}=A_{4} \mathbf{x}(t)+b_{4}$

The IT2 T-S fuzzy model is defined in Eq. (17) and the lower and upper membership functions are required to satisfy the inequalities (19) and (20).

Based on assumptions 1 and 2 and considering numerical values, it can be determined that the lower and upper membership functions of the IT2 T-S fuzzy model are the lower and upper bounds of $\mu_{\widetilde{M}_{1}^{i}}\left(Z_{1}(\mathbf{x}(t))\right)$ and $\mu_{\widetilde{M}_{2}^{i}}\left(Z_{2}(\mathbf{x}(t))\right)$. We can see that $Z_{1}(\mathbf{x}(t))$ has no uncertain parameter and therefore, lower and upper membership functions are the same. Table 2 shows the lower and upper membership functions based on sector nonlinearity. The lower and upper normalized grades of membership for each 
rule must satisfy Eq. (21). Plots of the lower and upper membership functions and FOU of the IT2 T-S fuzzy model are shown in Figs. 6 and 7.

Figure 8 illustrates that the difference between fuzzy model and original system with $\Delta a=-0.1, \Delta b=-0.5$; $\Delta a=-0.1, \Delta b=0.5 ; \quad \Delta a=0.1, \Delta b=-0.5 ;$ and $\Delta a=$ $0.1, \Delta b=0.5$ tends to zero for mentioned values of $\Delta a$ and $\Delta b$, i.e. the fuzzy model can represent the original system.
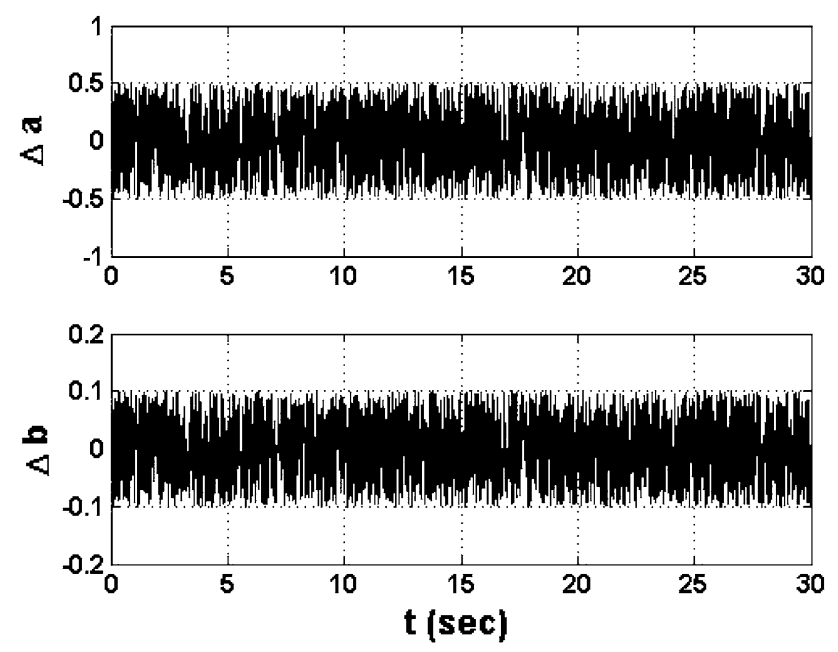

Fig. 5 White noises $\Delta a=0.1 \delta(t)$ and $\Delta b=0.5 \delta(t)$

Table 2 Lower and upper membership functions of Rossler system

\begin{tabular}{|c|c|}
\hline Lower membership functions & Upper membership functions \\
\hline$\underline{\mu}_{M_{1}^{1}}\left(Z_{1}(\mathbf{x}(t))\right)=\frac{10-x_{1}}{20}$ & $\bar{\mu}_{\widetilde{M}_{1}^{1}}\left(Z_{1}(\mathbf{x}(t))\right)=\frac{10-x_{1}}{20}$ \\
\hline$\underline{\mu}_{\widetilde{M}_{1}^{2}}\left(Z_{1}(\mathbf{x}(t))\right)=\frac{10-x_{1}}{20}$ & $\bar{\mu}_{\widetilde{M}_{1}^{2}}\left(Z_{1}(\mathbf{x}(t))\right)=\frac{10-x_{1}}{20}$ \\
\hline$\underline{\mu}_{\widetilde{M}_{1}^{3}}\left(Z_{1}(\mathbf{x}(t))\right)=\frac{10+x_{1}}{20}$ & $\bar{\mu}_{\widetilde{M}_{1}^{3}}\left(Z_{1}(\mathbf{x}(t))\right)=\frac{10+x_{1}}{20}$ \\
\hline$\underline{\mu}_{\widetilde{M}_{1}^{3}}\left(Z_{1}(\mathbf{x}(t))\right)=\frac{10+x_{1}}{20}$ & $\bar{\mu}_{\widetilde{M}_{1}^{4}}\left(Z_{1}(\mathbf{x}(t))\right)=\frac{10+x_{1}}{20}$ \\
\hline $\begin{array}{c}\mu_{M_{2}^{1}}\left(Z_{2}(\mathbf{x}(t))\right)=\frac{Z_{2 \max }-Z_{2}(\mathbf{x}(t))}{Z_{2 \max }-Z_{2 \min }} \\
\text { with }\left\{\begin{array}{l}\Delta a=0.1 \\
\Delta b=-0.5\end{array}\right.\end{array}$ & $\begin{array}{l}\bar{\mu} \widetilde{M}_{2}^{1}\left(Z_{2}(\mathbf{x}(t))\right)=\frac{Z_{2 \max }-Z_{2}(\mathbf{x}(t))}{Z_{2 \max }-Z_{2 \min }} \text { with } \\
\left\{\begin{array}{l}\Delta a=-0.1 \\
\Delta b=0.5\end{array}\right.\end{array}$ \\
\hline $\begin{array}{r}\mu_{\sim_{2}^{2}}\left(Z_{2}(\mathbf{x}(t))\right)=\frac{-Z_{2 \min }+Z_{2}(\mathbf{x}(t))}{Z_{2 \max }-Z_{2 \min }} \\
\text { with }\left\{\begin{array}{l}\Delta a=-0.1 \\
\Delta b=0.5\end{array}\right.\end{array}$ & $\begin{array}{l}\mu_{\widetilde{M}_{2}^{2}}\left(Z_{2}(\mathbf{x}(t))\right)=\frac{Z_{2 \max }+Z_{2}(\mathbf{x}(t))}{Z_{2 \max }-Z_{2 \min }} \text { with } \\
\left\{\begin{array}{l}\Delta a=0.1 \\
\Delta b=-0.5\end{array}\right.\end{array}$ \\
\hline $\begin{array}{c}\mu_{M_{2}^{3}}\left(Z_{2}(\mathbf{x}(t))\right)=\frac{Z_{2 \max }-Z_{2}(\mathbf{x}(t))}{Z_{2 \max }-Z_{2 \min }} \\
\text { with }\left\{\begin{array}{l}\Delta a=0.1 \\
\Delta b=-0.5\end{array}\right.\end{array}$ & $\begin{array}{l}\bar{\mu} \widetilde{M}_{2}^{3}\left(Z_{2}(\mathbf{x}(t))\right)=\frac{Z_{2 \max }-Z_{2}(\mathbf{x}(t))}{Z_{2 \max }-Z_{2 \min }} \text { with } \\
\left\{\begin{array}{l}\Delta a=-0.1 \\
\Delta b=0.5\end{array}\right.\end{array}$ \\
\hline $\begin{array}{c}\underline{\mu}_{M_{2}^{4}}\left(Z_{2}(\mathbf{x}(t))\right)=\frac{-Z_{2 \min }+Z_{2}(\mathbf{x}(t))}{Z_{2 \max }-Z_{2 \min }} \\
\text { with }\left\{\begin{array}{l}\Delta a=-0.1 \\
\Delta b=0.5\end{array}\right.\end{array}$ & $\begin{array}{l}\bar{\mu} \widetilde{M}_{2}\left(Z_{2}(\mathbf{x}(t))\right)=\frac{Z_{2 \max }+Z_{2}(\mathbf{x}(t))}{Z_{2 \max }-Z_{2 \min }} \text { with } \\
\left\{\begin{array}{l}\Delta a=0.1 \\
\Delta b=-0.5\end{array}\right.\end{array}$ \\
\hline
\end{tabular}

When membership functions are determined or tuned based on numerical data, the uncertainties in the numerical data translate into uncertainties in the membership functions as FOU, which is depicted in Figs. 6 and 7. In these figures, infinite number of type- 1 fuzzy model can be defined based on available FOU. So, the proposed IT2 T-S fuzzy model is a collection of type-1 T-S fuzzy models. Simulations show the difference between fuzzy model and original system of Rossler system with four values of uncertainties. As it is evident in Fig. 8, the modeling error or difference between fuzzy model and original system

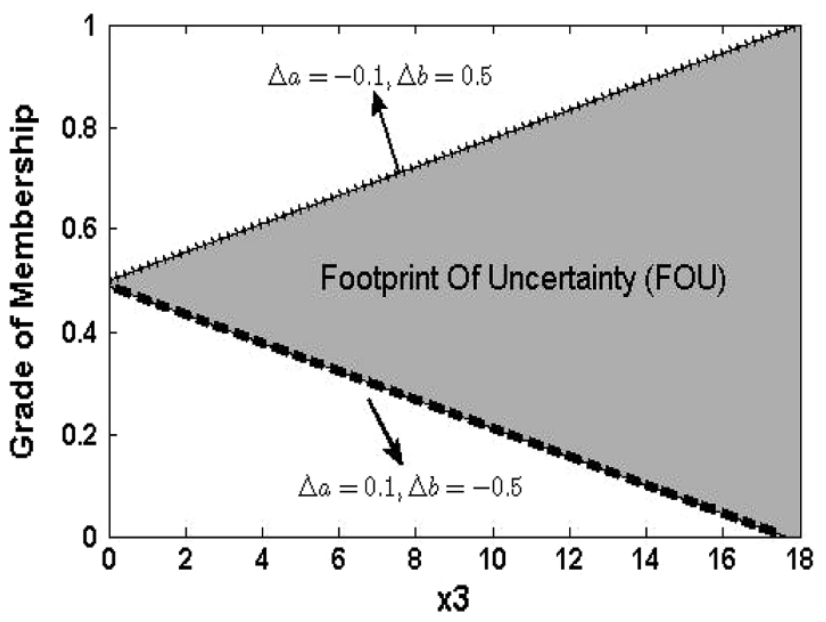

Fig. 6 Plot of $\mu_{\widetilde{M}_{2}^{1}}\left(Z_{2}(\mathbf{x}(t))\right)=\frac{Z_{2 \max }-Z_{2}(\mathbf{x}(t))}{Z_{2 \max }-Z_{2 \min }}$, lower membership function $\underline{\mu}_{\widetilde{M}_{2}^{1}}\left(Z_{2}(\mathbf{x}(t))\right)^{2}$ with $\Delta a=0.1$ and $\Delta b=-0.5$ (dash-dot line), upper membership function $\bar{\mu}_{\widetilde{M}_{2}^{1}}\left(Z_{2}(\mathbf{x}(t))\right)$ with $\Delta a=-0.1$ and $\Delta b=0.5$ (dotted line) and footprint of uncertainty (grey area)

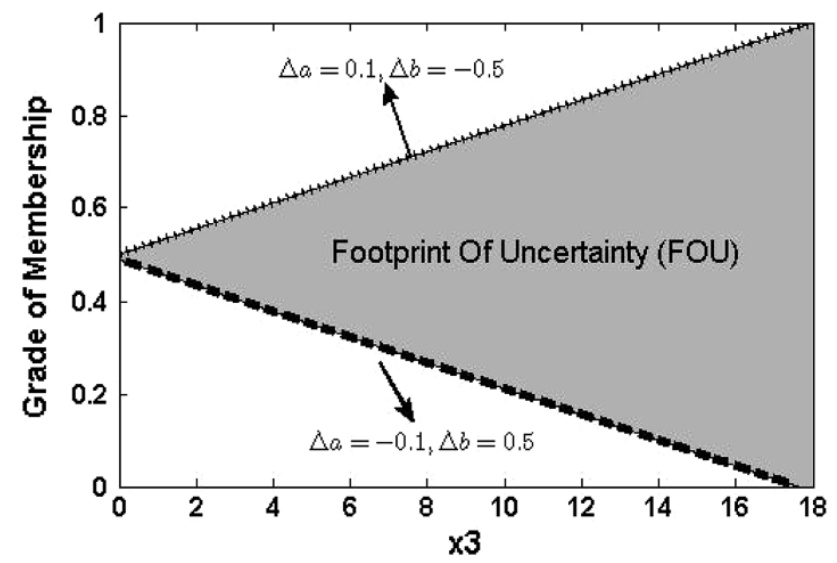

Fig. 7 Plot of $\mu_{\widetilde{M}_{2}^{2}}\left(Z_{2}(\mathbf{x}(t))\right)=\frac{Z_{2 \max }-Z_{2}(\mathbf{x}(t))}{Z_{2 \max }-Z_{2 \min }}$, lower membership function $\underline{\mu}_{\widetilde{M}_{2}^{2}}\left(Z_{2}(\mathbf{x}(t))\right)$ with $\Delta a=-0.1$ and $\Delta b=0.5$ (dash-dot line), upper membership function $\bar{\mu}_{\widetilde{M}_{2}^{2}}\left(Z_{2}(\mathbf{x}(t))\right)$ with $\Delta a=0.1$ and $\Delta b=-0.5$ (dotted line) and footprint of uncertainty (grey area) 

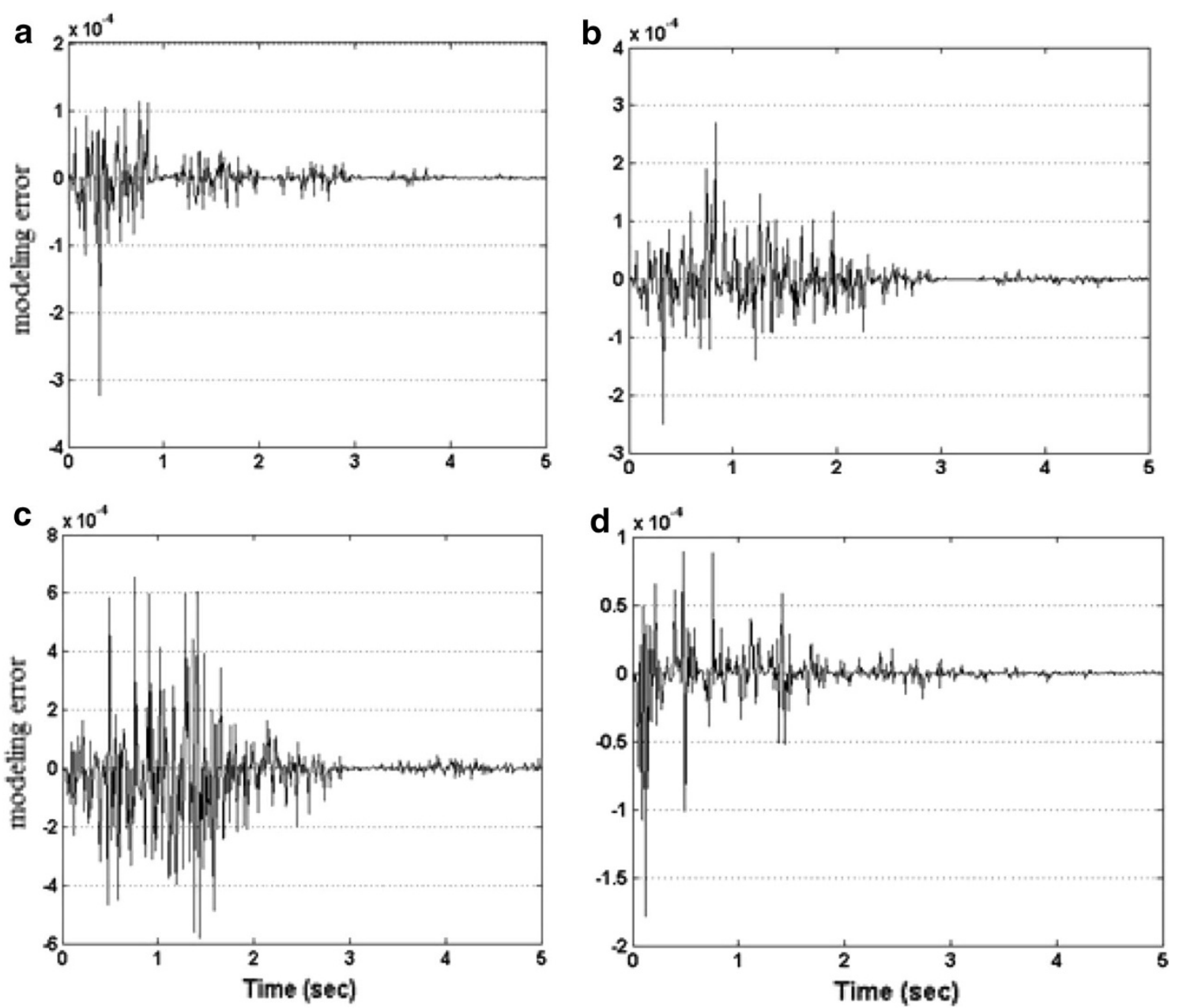

Fig. 8 Difference between fuzzy model and original system of Rossler system with a $\Delta a=-0.1, \Delta b=-0.5, \mathbf{b} \Delta a=-0.1, \Delta b=0.5$, c $\Delta a=0.1, \Delta b=-0.5$ and $\mathbf{d} \Delta a=0.1, \Delta b=0.5$

becomes less than $1.5 \times 10^{-4}$ after about $1.5 \mathrm{~s}$ which means that fuzzy model tends to original system.

The main advantage of this method of modeling is simplicity. In this method, if there are the lower and upper bounds of scheduling variable as an interval, an IT2 T-S fuzzy model can be defined. The lower and upper bounds of scheduling variable can be determined in chaos systems. So, this method can be extended for every chaotic and hyper-chaotic system with any uncertain parameters.

\section{Discussion}

In the proposed method, we have considered parameteric uncertainties based on assumption 2 for two chaotic systems with some candidate of parameter perturbations. As it is evident from figures, the fuzzy models can be used instead of original systems for several chaotic applications. However, there is no proof for robustness of proposed method; we have just surveyed the effect of uncertainties in modeling for several values of parameter uncertainties in simulations. For more information about robustness and future works, the papers in [43-45] can be cited to improve this work.

\section{Conclusion}

In this paper, a new method introduced for representing many well-known chaotic systems subjected to parametric uncertainty or structural variation of system via interval type-2 (IT2) T-S fuzzy model and besides lower and upper membership functions of the interval type-2 fuzzy sets was defined. For chaotic systems that evolve within a bounded region of the state space and parametric uncertainty, the IT2 T-S fuzzy model was appropriate for representing the nonlinear dynamics by lower and upper membership functions of interval type- 2 fuzzy sets. The proposed model 
can be used for chaotic control and synchronization. Numerically, the proposed method was applied to Lorenz and Rossler systems with one uncertain parameter and two uncertain parameters, respectively. It has been shown that type-1 fuzzy model cannot represent the chaos system with parametric uncertainty. The main advantage of proposed method is simplicity in mathematical computations and modeling parametric uncertainties.

Acknowledgments We would like to thank the editor and the referee for their timely and valuable comments.

Conflict of interest The authors declare that they have no competing interests.

Authors' contributions All calculations, simulations of the various results and their verifications were carried out under MATLAB environment. All authors read and approved the final manuscript.

Open Access This article is distributed under the terms of the Creative Commons Attribution License which permits any use, distribution, and reproduction in any medium, provided the original author(s) and the source are credited.

\section{References}

1. Alligood, K.T., Sauer, T., Yorke, J.A.: Chaos: An Introduction to Dynamical Systems. Springer, New York (1997)

2. Vincent, U.E., Njah, A.N., Akinlade, O., Solarin, A.R.T.: Phase synchronization in unidirectional coupled chaotic ratchets. Chaos 14, 1018-1025 (2004)

3. Vincent, U.E., Njah, A.N., Akinlade, O., Solarin, A.R.T.: Phase synchronization in bidirectional coupled chaotic ratchets. Phys. A 360, 180-196 (2006)

4. Lakshmanan, M., Murali, K.: Nonlinear Oscillators: Controlling and Synchronization. World Scientific, Singapore (1996)

5. Niu, H., Zhang, Q., Zhang, Y.: The chaos synchronization of a singular chemical model and a Williamowski-Rossler model. Int. J. Inform. Syst. Sci. 6(4), 355-364 (2002)

6. Kocarev, L., Parlitz, U.: General approach for chaotic synchronization with applications to communication. Phys. Rev. Lett. 74, 5028-5030 (1995)

7. Boccaletti, S., Farini, A., Arecchi, F.T.: Adaptive synchronization of chaos for secure communication. Phys. Rev. E 55, 5 (1997)

8. Tao, Y.: Chaotic secure communication systems-history and new results. Telecommun. Rev. 9, 597-634 (1999)

9. Zhang, H.G., Yang, D.D., Chai, T.Y.: Guaranteed cost networked control for T-S fuzzy systems with time delay. IEEE Trans Syst Man Cybern C 37, 160-172 (2007)

10. Zhang, H.B., Liao, X.F., Yu, J.B.: Fuzzy modeling and synchronization of hyper chaotic systems. Chaos Solitons Fractals 26, 835-843 (2005)

11. Lian, K.Y., Chiang, T.S., Chiu, C.S., Liu, P.: Synthesis of fuzzy model-based designs to synchronization and secure communications for chaotic systems. IEEE Trans. Syst. Man Cybern. 31, 66-83 (2001)

12. Takagi, T., Sugeno, M.: Fuzzy identification of systems and its applications to modeling and control. IEEE Trans. Syst. Man Cybern. 15, 116-132 (1985)

13. Yang, D.S., Zhang, H.G., Li, H.G., Meng, Z.Y.: Generalized synchronization of two non identical chaotic systems based on fuzzy model. Acta Phys. Sinica 56, 3121-3126 (2007)
14. Lam, H.K., Leung, F.H.F.: Synchronization of uncertain chaotic systems based on the fuzzy-model-based approach. Int. J. Bifurc. Chaos 16, 1435-1444 (2006)

15. Lian, K.Y., LIU, P., Wu, T.C., Lin, W.C.: Chaotic control using fuzzy model-based methods. Int. J. Bifurc. Chaos 12, 1827-1841 (2002)

16. Pourkargar, D.B., Shahrokhi, M.: Optimal fuzzy synchronization of generalized lorenz chaotic system. TJMCS 2, 27-36 (2011)

17. Hong, L., Fei, L., Zhong, L., Xiajie, Y., Trillion, Q., Zhang, B.: The application of chaotic PWM control for EMI suppression. COMPEL Int. J. Comput. Math. Electr. Electron. Eng 32, 750-762 (2013)

18. Wu, D., Tan, W.W.: Genetic learning and performance evaluation of type-2 fuzzy logic controllers. Eng. Appl. Artif. Intell. 19, 829-841 (2006)

19. Hagras, H.: A hierarchical type-2 fuzzy logic control architecture for autonomous mobile robots. IEEE Trans. Fuzzy Syst. 12, 524-539 (2004)

20. Hagras, H.: Type-2 FLCs: a new generation of fuzzy controllers. IEEE Comput. Intell. Mag. 2, 30-43 (2007)

21. Mendel, J.M.: Uncertain rule-based fuzzy logic systems: introduction and new directions, upper saddle river. Prentice-Hall, NJ (2001)

22. Jammeh, E.A., Fleury, M.C., Hagras, H., Ghanbari, M.: Interval type-2 fuzzy logic congestion control for video streaming across IP networks. IEEE Trans. Fuzzy Syst. 17, 1123-1142 (2009)

23. Mendel, J., John, R.I., Liu, F.: Interval type-2 fuzzy logic systems made simple. IEEE Trans. Fuzzy Syst. 14, 808-821 (2006)

24. Mendel, J., Liu, F.: Super-exponential convergence of the Karnik-Mendel algorithms for computing the centroid of an interval type-2 fuzzy set. IEEE Trans. Fuzzy Syst. 15, 309-320 (2007)

25. Roopaei, M., Zolghadri, M.B., Lin, T.C.: Synchronization of two different chaotic systems using novel adaptive interval type2 fuzzy sliding mode control. Nonlinear Dyn. 66, 667-680 (2011)

26. Coupland, S., John, R.: Geometric type-1 and type-2 fuzzy logic systems. IEEE Trans. Fuzzy Syst. 15, 3-15 (2007)

27. Leal Ramirez, C., Castillo, O., Melin, P., Rodriguez Diaz, A.: Simulation of the bird age-structured population growth based on an interval type-2 fuzzy cellular structure. Inf. Sci. 181(3), 519-535 (2011)

28. Melin, P., Astudillo, L., Castillo, O., Valdez, F., Garcia, M.: Optimal design of type-2 and type-1 fuzzy tracking controllers for autonomous mobile robots under perturbed torques using a new chemical optimization paradigm. Expert Syst. Appl. 40(8), 3185-3195 (2013)

29. Castillo, O., Melin, P.: A review on the design and optimization of interval type-2 fuzzy controllers. Appl. Soft. Comput. 12(4), 1267-1278 (2012)

30. Hidalgo, D., Castillo, O., Melin, P.: Type-1 and type-2 fuzzy inference systems as integration methods in modular neural networks for multimodal biometry and its optimization with genetic algorithms. Inf. Sci. 179(13), 2123-2145 (2009)

31. Wu, D., Tan, W.W.: A simplified type-2 fuzzy controller for realtime control. ISA Trans. 15, 503-516 (2006)

32. $\mathrm{Wu}, \mathrm{D} .:$ On the fundamental differences between interval type-2 and type-1 fuzzy logic controllers, IEEE Trans. Fuzzy Syst., in press (2012)

33. Castillo, O., Melin, P.: Type-2 Fuzzy Logic Theory and Application. Springer, Berlin (2008)

34. Du, X., Ying, H., Lin, F.: On modeling of fuzzy hybrid systems. J. Intell. Fuzzy Syst. 23, 129-141 (2012)

35. Hénon, M.: A two-dimensional mapping with a strange attractor. Commun. Math. Phys. 50(1), 69-77 (1976)

36. Rössler, O.E.: An equation for continuous Chaos. Phys. Lett. 57A(5), 397-398 (1976) 
37. Lorenz, E.N.: Deterministic nonperiodic flow. J. Atmos. Sci. 20(2), 130-141 (1963)

38. Bunch, J.R., Hopcroft, J.: Triangular factorization and inversion by fast matrix multiplication. Math. Comput. 28, 231-236 (1974)

39. Huangzhang, Liu, D., Wang, Z.: Controlling Chaos, Suppression, Synchronization and Chaotification. Springer, Berlin (2009)

40. Lam, H.K., Leung, F.: Stability analysis of fuzzy model based control systems. Springer 264, 191-215 (2011)

41. Liang, Q., Mendel, J.M.: Equalization of nonlinear time-varying channels using type-2 fuzzy adaptive filters. IEEE Trans. Fuzzy Syst. 8(5), 551-563 (2000)

42. Ohtake, H., Tanaka, K., Wang, H.: Fuzzy modeling via sector nonlinearity concept. In: Proceedings of the Joint 9th IFSA World Congress and 20th NAFIPS International Conference, Vancouver, Canada 1, 127-132 (2001)
43. Qiu, J., Feng, G., Yang, J.: A new design of delay-dependent robust $\mathrm{H}$-infinity filtering for discrete-time T-S fuzzy systems with time-varying delay. IEEE Trans. Fuzzy Syst. 17(5), 1044-1058 (2009)

44. Qiu, J., Feng, G., Gao, H.: Fuzzy-model-based piecewise $\mathrm{H}$-infinity static output feedback controller design for networked nonlinear systems". IEEE Trans. Fuzzy Syst. 18(5), 919-934 (2010)

45. Qiu, J., Feng, G., Gao, H.: Static output feedback H-infinity control of continuous-time T-S fuzzy affine systems via piecewise Lyapunov functions. IEEE Trans. Fuzzy Syst. 21(2), 245-261 (2013) 